\title{
Response of Assorted Maize Germplasm to the Maize Lethal Necrosis Disease in Kenya
}

\author{
Sitta, J. ${ }^{1}$, Nzuve, F. M. ${ }^{2}$, Olubayo, F. M. ${ }^{2}$, Mutinda, C. ${ }^{3}$, Muiru, W. M. ${ }^{2}$, Miano, D. W. ${ }^{2}$, Muthomi, J. W. ${ }^{2}$ \& \\ Leley, P. K. ${ }^{3}$ \\ ${ }^{1}$ Chollima Agro-Scientific Research Centre, P. O. Box 1892, Morogoro, Tanzania \\ ${ }^{2}$ Department of plant science and Crop Protection, University of Nairobi, P O Box 29053-00625, Nairobi, Kenya \\ ${ }^{3}$ Kenya Agricultural and Livestock Research Organization, P O Box 57811-00200, Kenya \\ Correspondence: Felister Nzuve, University of Nairobi, Kenya. E-mail: fmbute@yahoo.com
}

Received: February 25, 2017 Accepted: May 9, $2017 \quad$ Online Published: July 16, 2017

doi:10.5539/jps.v6n2p65

URL: https://doi.org/10.5539/jps.v6n2p65

\begin{abstract}
Maize (Zea mays L.) is the most widely grown staple food crop in Sub Saharan Africa (SSA) and occupies more than 33 million hectares each year. The recent outbreak and rapid spread of the Maize Lethal Necrosis (MLN) disease has emerged as a great challenge to maize production, threatening food security for the majority of households in the Eastern Africa region with yield loss estimated to be 50-90\%. The disease is a result of synergistic interaction between two viruses, Sugarcane mosaic virus (SCMV) and Maize chlorotic mottle virus (MCMV). The objective of this study was to identify maize genotypes with resistance to MLN. In season one, 73 maize genotypes comprising 25 inbred lines from research institutes, 30 lines from the International Maize and Wheat Improvement Centre (CIMMYT) and 18 farmer varieties were screened for resistance to MLN. In season 2, only 48 genotypes were screened after some of the inbred lines showed complete susceptibility to MLN. These genotypes were grown in three replications in a completely randomized design in polythene bags in the greenhouse at the University of Nairobi. The plants were artificially inoculated using a mixture of SCMV and MCMV. .Weekly MLN disease severity scores using a scale of 1 to $5(1=$ highly resistant and $5=$ highly susceptible) and \% MLN incidence were recorded and eventually converted into Area under Disease Progress Curve (AUDPC) to give an indication of the disease intensity over time. The plants were allowed to grow to flowering stage to observe the effect of the MLN on the maize productivity. Analysis of Variance revealed wide genetic variation among the genotypes ranging from resistant to highly susceptible. In season 1, three farmer varieties namely MLR2, MLR11 and MLR13 showed resistance to MLN with a mean severity score of 2. In season 2, MLN12, MLN17, MLN18, MLN19, and MLR4 showed low MLN severity ranging from 2-3. The genotypes MLR6, MLR9, MLR16 and MLR18 showed MLN severity of 3 and early maturity traits. This study also validated the presence of MLN resistance among some CIMMYT lines depicted to show resistance in previous studies. These resistant genotypes could serve as donors in the introgression of the resistance into the adapted Kenyan maize backgrounds. This will go a long way in ensuring sustainable maize productivity while improving the livelihoods of the small-scale farmers who form the bulk of the major maize producers in Kenya.
\end{abstract}

Keywords: maize lethal necrosis disease, MLN severity, maize genotypes

\section{Introduction}

In Kenya, food security is synonymous with maize availability since it is a key staple food to over $90 \%$ of her population with about 42 dietary energy intakes (Keya and Rubaihayo, 2013). The recent outbreak and rapid spread of the Maize Lethal Necrosis (MLN) disease has emerged as a great challenge to maize production, threatening food security for the majority of households in the region. Maize lethal necrosis is a serious disease of maize, and since its first appearance in Kenya in 2011 (Wangai et al., 2012), the disease has spread to many countries in the East Africa region where maize is grown including Tanzania, Uganda, South Sudan and Rwanda (IITA, 2014). The disease has been identified as the most devastating foliar disease responsible for highest yield loss in maize because it causes the yield loss of up to $100 \%$ because of its ability to kill infected plant and cells (Mbega et al., 2016).

MLN is caused by a mixed or synergistic infection between Maize chlorotic mottle virus (MCMV, genus Machlomovirus) and potyviruses infecting maize which includes either Sugarcane mosaic virus (SCMV) or 
Maize dwarf mosaic virus (MDMV) and Wheat streak mosaic virus (WSMV). The synergism between viruses refers to situations where co-infection with two viruses leads to more virulence as opposed to singular infections. It is also a state where multiplication of at least one of the viruses is enhanced by the other (Shi et al., 1997; Karyeija et al., 2000). The MCMV belongs to the genus Machlomovirus of the family Tombusviridae. It was first identified in maize in Peru in 1974 and USA in 1978 and 1980 (Wu et al., 2013). It is most common in the graminae family. The SCMV causes mosaic diseases and consists of four distinct Potyviruses including strains of Johnsongrass mosaic virus (JGMV), MDMV, Sorghum mosaic virus (SrMV) and SCMV (Yang and Mirkov, 1997). The MCMV causes mild mosaic, severe stunting, leaf necrosis, premature plant death, shortened male inflorescences with few spikes, and shortened, malformed and partially filled ears (Wu et al., 2013). Sugarcane mosaic virus and MDMV are two important pathogens of maize and related crops, causing yield losses, chlorosis and stunting (Xu et al., 2000). The infected maize plants are frequently barren, the ears formed are small, deformed and set little or no seeds, drastically reducing the yield. This greatly affects the physiological processes like photosynthesis and chlorophyll formation (Wangai et al., 2012), causing failure of tasseling or sterility in male plants. These could also lead to deformed or no ears or even rotting of the cobs (Adams et al., 2013).The MLN disease also predisposes the plants to secondary fungal infections (FSNWG, 2012; Wu et al., 2013). The type of virus, plant phenology, time of infection, host plant growth conditions and genotype all influence the extent of disease spread and distribution (Melchinger et al., 1998). The disease is also aggravated by drought conditions and poor soil fertility (http://www.fao.org/2012). Other predisposing factors include the growing of host crops like sugarcane and millet that contributes towards increased inoculum loads. Insect vectors also aid in the transmission of viruses e.g. maize thrips and beetles have been associated with the transmission of MCMV while aphids transmit SCMV (Cabanas et al., 2013; Wu et al., 2013). Other forms of transmission include either mechanical or through the seed (Miano and Kabaki, 2013; Wu et al., 2013).

The MLN challenge is compounded by the fact that the viruses inhabit leaves, pollen, female and male inflorescences, ear husks, cotyledons and seeds (Nelson et al., 2011) further complicating MLN disease management. A gene GRMZM2G018943 was reported to function as the translation initiation factor eIF-2B and this was associated with the mutation of plant proteins which aims at countering the viral attack (Ingvardsen $e t$ al., 2010). However, this seems not to be the case especially among the currently grown maize varieties in Kenya which have shown high susceptibility to MLN. The nature of entry and replication of the SCMV virus into the plants also aggravates the MLN menace (Ingvardsen et al., 2010; Gowda et al., 2015). Knowledge of the virus dynamics could offer a more reliable approach towards management of the MLN epidemics (Redinbaugh et al., 2000). The management of MLN disease could be achieved through integrating cultural methods, chemical method such as seed dressing and foliar spray with host resistance breeding. In Kenya, poor agricultural practices like leaving infected maize crop residues in the field and maize monoculture do not aid in breaking the disease transmission cycle. This implies that insect vectors transmit the viruses through crops and seasons. Lack of proper weed management practices has also aided in offering alternative hosts to these vectors since these weeds are susceptible to the MCMV. The use of chemicals is uneconomical and environmentally unfriendly especially among the resource constrained small-scale maize farmers. These chemicals are rendered ineffective due to the non-persistent transmission of the viruses (Melchinger et al., 1998).

Host breeding for resistance is the most ecologically safe and economical approach towards combating the MLN disease menace. Previous breeding efforts by the International Maize and Wheat Improvement Centre (CIMMYT) and Kenya Agricultural and Livestock Research Organization (KALRO) have reported very high susceptibility of around 90\% to MLN disease among the pre-commercial and commercial maize germplasm (Gowda et al., 2015). This implies that reduction in maize production due to the impact of MLN disease will and has already adversely affected the Kenyan livelihoods and the overall market prices of maize (Wangai et al., 2012). The areas affected constitute major maize production acreage and given the recorded loss of up to $100 \%$, it has become an important food security issue in Kenya. The impact of the disease has been felt in the whole maize value chain.

Efforts put in breeding to improve maize productivity are aimed at developing many varieties which are resistant to both biotic and abiotic stresses. CIMMYT has undertaken discovery studies to identify genomic regions associated with MLN disease resistance where two mapping panels and six bi-parental populations have revealed three major QTLs on chromosomes and a few minor QTLs across other chromosomes (Olsen et al., 2016). These QTLs are aimed for introgression into adapted maize genotypes. The identification of new sources of resistance could contribute valuable alleles which may supplement the ones in use. The objective of this study was to determine the response of assorted maize genotypes to MLN infection and identify any heterotic parents which could be utilized in maize breeding programs to improve maize productivity in the region. 


\section{Materials and Methods}

\subsection{Germplasms}

The maize genotypes were assembled from diverse sources namely CIMMYT, KALRO and farmers' varieties (landraces) (Table 1).

\subsection{Experiment Site Description}

The experiments were done in the greenhouse located at the University of Nairobi Field Station (Kabete) for two seasons. Kabete is situated at Latitude of $1^{\circ} 15^{\prime} \mathrm{S}$ and Longitude $36^{\circ} 44^{\prime} \mathrm{E}$, and at an altitude $1930 \mathrm{~m}$ above sea level. Soils are usually humic nitisol, well drained, deep $(>180 \mathrm{~cm})$, dark red to darkish brown. Kabete has a bimodal distribution of rainfall, with long rains from early March to late May and the short rains from October to December. The mean annual temperature is $18^{\circ} \mathrm{C}$ and the mean annual rainfall is $1006 \mathrm{~mm}$ (Onyango et al., 2012).

\subsubsection{Experiment Design and Layout}

The experiment was set up in a completely randomized design in three replications. The genotypes were grown in polythene bags measuring $20 \mathrm{~cm}$ in diameter in the greenhouse. Previous studies have validated the use of greenhouse due to the fact that the plants are tender leading to efficacy in inoculations and clear genotype diagnosis (Melchinger et al., 1998). All the agronomic practices were observed namely; hand weeding, irrigation, fertilizer application whereby DAP was used during planting at the rate of $10 \mathrm{~g} / \mathrm{hill}$ while urea was used as a top dressing fertilizer. After 14 days post germination, the genotypes were artificially inoculated using a combination of both viruses namely MCMV and SCMV through hand rubbing.

\subsubsection{Isolation of the Pathogen}

The MLN causal viruses namely MCMV SCMV were isolated from diseased tissue of maize leaves showing clear virus symptoms at KALRO where the two viruses are maintained at the Biosafety Greenhouse (BGH).

\subsubsection{Preparation of Inocula and Inoculation}

The leaves were cut into small pieces and stored in the freezer at a temperature of $-20^{\circ} \mathrm{C}$. Phosphate buffer 0.1 $\mathrm{M}$ was made by mixing potassium phosphate Dibasic (Anhydrous) and Potassium dihydrogen orthophosphate (Potassium phosphate monobasic) to a $\mathrm{pH}$ of 7.0 using the following ratios; $\mathrm{KH}_{2} \mathrm{PO} 4=10.8 \mathrm{~g}, \mathrm{~K}_{2} \mathrm{HPO} 4=4.8 \mathrm{~g}$ and $\mathrm{Na}_{2} \mathrm{SO} 3=1.26$ and Carborandum $(\mathrm{SiCO} 3)=1 \mathrm{~g} / \mathrm{l}$. Then $2 \mathrm{~g}$ of leaves with MCMV and $10 \mathrm{~g}$ of leaves with SCMV at a ratio of 1:5 were weighed and ground using sterile mortar and pestle to obtain homogenate solution or extract. The extract was added to the buffer to make $120 \mathrm{ml}$. The combination of MCMV and SCMV inoculums was then rubbed onto two week old young leaves of the maize seedlings in the greenhouse. The Carborandum powder $\left(\mathrm{SiCO}_{3}\right)$ which is an abrasive agent was used to cause microscopic injury of the leaves for easy penetration of the virus into the plant cells (Orawu et al., 2013). A second inoculation was done at the interval of one week from the first inoculation to ensure effective viral dissemination and spread among the genotypes and that there were no diseases escapes.

Table 1. List of the genotypes used for screening and their sources

\begin{tabular}{lllll}
\hline UoN Designation & Genotype & Origin & Season 1 & Season 2 \\
\hline UoN-2015-51 & KAT25-1 & KALRO & 1 & - \\
UoN-2015-52 & KAT25-2 & KALRO & 1 & - \\
UoN-2015-53 & KAT25-3 & KALRO & 1 & - \\
UoN-2015-54 & KAT25-4 & KALRO & 1 & - \\
UoN-2015-55 & KAT25-5 & KALRO & 1 & - \\
UoN-2015-56 & KAT25-6 & KALRO & 1 & - \\
UoN-2015-57 & KAT25-7 & KALRO & 1 & - \\
UoN-2015-58 & KAT25-8 & KALRO & 1 & - \\
UoN-2015-59 & KAT25-9 & KALRO & 1 & - \\
UoN-2015-60 & KAT25-10 & KALRO & 1 & - \\
UoN-2015-61 & KAT25-11 & KALRO & 1 & - \\
UoN-2015-62 & KAT25-12 & KALRO & 1 & - \\
UoN-2015-63 & KAT25-13 & KALRO & 1 & - \\
UoN-2015-64 & KAT25-14 & KALRO & 1 & - \\
UoN-2015-65 & KAT25-15 & KALRO & 1 & - \\
UoN-2015-66 & KAT25-16 & KALRO & 1 & - \\
UoN-2015-67 & KAT25-17 & KALRO & 1 & - \\
UoN-2015-68 & KAT25-18 & KALRO & 1 & - \\
UoN-2015-69 & KAT25-19 & KALRO & 1 & - \\
UoN-2015-70 & KAT25-20 & KALRO & 1 & - \\
\hline
\end{tabular}




\begin{tabular}{|c|c|c|c|c|}
\hline UoN Designation & Genotype & Origin & Season 1 & Season 2 \\
\hline UoN-2015-71 & KAT25-21 & KALRO & 1 & - \\
\hline UoN-2015-72 & KAT25-22 & KALRO & 1 & - \\
\hline UoN-2015-73 & KAT25-23 & KALRO & 1 & - \\
\hline UoN-2015-74 & KAT25-24 & KALRO & 1 & - \\
\hline UoN-2015-75 & KAT25-25 & KALRO & 1 & - \\
\hline UoN-2015-76 & MLN1 & CIMMYT & 1 & 2 \\
\hline UoN-2015-77 & MLN2 & CIMMYT & 1 & 2 \\
\hline UoN-2015-78 & MLN3 & CIMMYT & 1 & 2 \\
\hline UoN-2015-79 & MLN4 & CIMMYT & 1 & 2 \\
\hline UoN-2015-80 & MLN5 & CIMMYT & 1 & 2 \\
\hline UoN-2015-81 & MLN6 & CIMMYT & 1 & 2 \\
\hline UoN-2015-82 & MLN7 & CIMMYT & 1 & 2 \\
\hline UoN-2015-83 & MLN8 & CIMMYT & 1 & 2 \\
\hline UoN-2015-84 & MLN9 & CIMMYT & 1 & 2 \\
\hline UoN-2015-85 & MLN10 & CIMMYT & 1 & 2 \\
\hline UoN-2015-86 & MLN11 & CIMMYT & 1 & 2 \\
\hline UoN-2015-87 & MLN12 & CIMMYT & 1 & 2 \\
\hline UoN-2015-88 & MLN13 & CIMMYT & 1 & 2 \\
\hline UoN-2015-89 & MLN14 & CIMMYT & 1 & 2 \\
\hline UoN-2015-90 & MLN15 & CIMMYT & 1 & 2 \\
\hline UoN-2015-91 & MLN16 & CIMMYT & 1 & 2 \\
\hline UoN-2015-92 & MLN17 & CIMMYT & 1 & 2 \\
\hline UoN-2015-93 & MLN18 & CIMMYT & 1 & 2 \\
\hline UoN-2015-94 & MLN19 & CIMMYT & 1 & 2 \\
\hline UoN-2015-95 & MLN20 & CIMMYT & 1 & 2 \\
\hline UoN-2015-96 & MLN21 & CIMMYT & 1 & 2 \\
\hline UoN-2015-97 & MLN22 & CIMMYT & 1 & 2 \\
\hline UoN-2015-98 & MLN23 & CIMMYT & 1 & 2 \\
\hline UoN-2015-99 & MLN24 & CIMMYT & 1 & 2 \\
\hline UoN-2015-100 & MLN25 & CIMMYT & 1 & 2 \\
\hline UoN-2015-101 & MLN26 & CIMMYT & 1 & 2 \\
\hline UoN-2015-102 & MLN27 & CIMMYT & 1 & 2 \\
\hline UoN-2015-103 & MLN28 & CIMMYT & 1 & 2 \\
\hline UoN-2015-104 & MLN29 & CIMMYT & 1 & 2 \\
\hline UoN-2015-105 & MLN30 & СIMMYT & 1 & 2 \\
\hline UoN-2015-106 & MLR1 & Farmer varieties & 1 & 2 \\
\hline UoN-2015-107 & MLR2 & Farmer varieties & 1 & 2 \\
\hline UoN-2015-108 & MLR3 & Farmer varieties & 1 & 2 \\
\hline UoN-2015-109 & MLR4 & Farmer varieties & 1 & 2 \\
\hline UoN-2015-110 & MLR5 & Farmer varieties & 1 & 2 \\
\hline UoN-2015-111 & MLR6 & Farmer varieties & 1 & 2 \\
\hline UoN-2015-112 & MLR7 & Farmer varieties & 1 & 2 \\
\hline UoN-2015-113 & MLR8 & Farmer varieties & 1 & 2 \\
\hline UoN-2015-114 & MLR9 & Farmer varieties & 1 & 2 \\
\hline UoN-2015-115 & MLR10 & Farmer varieties & 1 & 2 \\
\hline UoN-2015-116 & MLR11 & Farmer varieties & 1 & 2 \\
\hline UoN-2015-117 & MLR12 & Farmer varieties & 1 & 2 \\
\hline UoN-2015-118 & MLR13 & Farmer varieties & 1 & 2 \\
\hline UoN-2015-119 & MLR14 & Farmer varieties & 1 & 2 \\
\hline UoN-2015-120 & MLR15 & Farmer varieties & 1 & 2 \\
\hline UoN-2015-121 & MLR16 & Farmer varieties & 1 & 2 \\
\hline UoN-2015-122 & MLR17 & Farmer varieties & 1 & 2 \\
\hline
\end{tabular}

UoN= University of Nairobi; KALRO=Kenya Agricultural and Livestock Research Organization; CIMMYT=International Maize and Wheat Improvement Centre; - = not evaluated in season two

\subsection{Data Collection}

The MLN symptoms were assessed for disease severity based on the CIMMYT scale (Table 2) and percent MLN incidence. Disease severity scoring began one week after the repeat inoculation and this was done weekly for eight weeks. The delayed scoring for the presence of MLN was to detect late developing infections (Zambrano et al., 2013). The plants were allowed to grow to physiological maturity to enable one to get an indication of the effect of MLN on maturity and yielding potential. The percentage MLN incidence was measured as the percentage of the number of leaves with MLN infection. 
Table 1. CIMMYT scale used in assessment of the MLN disease

\begin{tabular}{ll}
\hline Score & Symptoms \\
\hline 1 & No MLN symptoms \\
2 & Fine chlorotic streaks \\
3 & Chlorotic mottling \\
4 & Excessive chlorotic mottling and some necrosis \\
5 & Dead heart symptoms/ complete plant death \\
\hline
\end{tabular}

Source (CIMMYT).

\subsection{Data Analysis}

The disease severity scores were converted into the Area Under Disease Progress Curve (AUDPC) values based on Wilcoxson et al. (1975). AUDPC is simply the intensity of disease integrated between two times. It is a crucial quantitative summary of the disease intensity over time for comparison across years, locations as well as management tactics. The AUDPC expresses the dynamics of an epidemic as a single value and different epidemics can be compared by normalizing the different AUDPC value of each epidemic by calculating the relative area under disease progress curve (AUDPC) (Equation (1) (Wilcoxson et al., 1975).

$$
\text { AUDPC }=\sum_{i=1}^{n-1}\left(\frac{y n+y n+1}{n}\right)(t i+1-t i) \quad \text { Equation } 1
$$

Whereby,

$\mathrm{n}=$ total number of observations, $\mathrm{y}_{i}=$ injury intensity (usually incidence in crop health data) at the $i$ th observation, $t=$ time at the $i$ th observation.

\subsubsection{Analysis of Variance}

The AUDPC values, the weekly MLN severity scores, \% MLN incidence and other agronomic traits were subjected to the analysis of variance (ANOVA). The genotype means were separated using Fisher's protected least significant differences (LSD) test at $5 \%$ significance level.

\subsubsection{Correlation among the Different Traits}

A Pearson's Correlation Coefficient to establish the phenotypic relationship among the different disease parameters assessed was done following the formula by Pearson (1896) shown on Equation (2).

$$
r=\frac{2[A-x][x-x]}{\longleftarrow}
$$

Where,

$\bar{X}=$ mean of $\mathrm{X}$ variable; $\bar{Y}=$ mean of $\mathrm{Y}$ variable

\section{Results}

\subsection{Analysis of Variance}

The findings revealed that there were significant differences among the genotypes for the different MLN disease parameters at $\mathrm{P}<0.05$. The replications were not significantly different implying that these findings were repeatable and reproducible through seasons (Table 3 and Table 4).

\subsection{Mean Performance of the Assorted Maize Germplasm across Two Seasons}

The mean performance of the maize genotypes under MLN artificial infection varied significantly across the two seasons (Table 5). During season 1, 73 genotypes were screened for resistance to MLN whereas in season 2, 50 genotypes were assessed for MLN resistance. The response of the genotypes to the MLN was assessed based on the parameters AUDPC, final disease severity (FS) and MLN disease incidence. To obtain absolute values, there was the use of the relative Final MLN severity (rFS) scores and relative Area Under Disease Progress Curve (rAUDPC).

The genotypes assembled from CIMMYT showed good resistance to the MLN especially during season 2 despite the heavy MLN disease pressure. The genotypes were also divergent with respect to either earliness or lateness in maturity with most of the KALRO and farmer varieties showing the earliness trait. Most of the CIMMYT derived genotypes showed lateness trait.

In season 1, the MLN severity scores ranged from 1 to 5 among the genotypes. The AUDPC had a range of 76 to 148 while the \% MLN incidence had a range of 5 to 100\%. During season 2, the MLN severity scores ranged from 2 to 5 among the genotypes. The AUDPC had a range of 109 to 246 while the \% MLN incidence had a 
range of 26 to $100 \%$.

In season one, twenty of the genotypes which showed high MLN resistance also had earliness trait namely KAT25-10, KAT25-16, MLN1, MLN15, MLN16, MLR-10, MLR-13 and MLR-17 with MLN scores ranging from 1 to 2 (Table 4). In season 2, MLN17, MLN19, MLR4, MLN18 and MLN12 combined good MLN disease parameters with MLN severity ranging from 2-3 and also statistically low AUDPC, rAUDPC and \% MLN incidence values. These genotypes also exhibited lateness in flowering with statistically many days to $50 \%$ tasseling and silking. The genotypes MLR6, MLR9, MLR16 and MLR18 showed MLN severity of 3 and early maturity with statistically few number of days to both $50 \%$ tasseling and silking.

\subsection{Correlations among Traits in Season across the Two Seasons}

The MLN disease parameters namely AUDPC, FS and \% DI showed positive and highly significant correlation coefficient values validating their use in estimating the most susceptible and most MLN resistant genotype (Table 7). The weekly MLN progressions also showed positive and highly significant correlation coefficients implying that the period of MLN evaluation was sufficient. However, no distinct relationship was established between the maturity indicators namely days to 50\% pollen shed and silking and the MLN assessment parameters.

Table 3. Mean Squares for the MLN disease parameters during season 1

\begin{tabular}{lllllll}
\hline Source of variation & df & \% incidence & MLN severity & AUDPC & rAUDPC & rFS \\
\hline Replications & 2 & 4444.90 & 3.90 & 2316.70 & 882.70 & 3.90 \\
Genotypes & 71 & $1879.4^{*}$ & $1.5^{*}$ & $993.3^{*}$ & $378.5^{*}$ & $1.5^{*}$ \\
Residual & 124 & 846.90 & 0.50 & 380.60 & 145.00 & 0.50 \\
Total & 197 & & & & & \\
\hline
\end{tabular}

Table 4. Mean squares for the MLN disease parameters during season 2

\begin{tabular}{lllllll}
\hline Source of variation & df & \% MLN incidence & MLN severity & AUDPC & rAUDPC & rFS \\
\hline Replications & 2 & 24240.80 & 8.27 & 46273.00 & 5210.70 & 17.33 \\
Entry & 46 & $10562.7^{*}$ & $5.43^{*}$ & $21743^{*}$ & $2448.4^{*}$ & $6.3 *$ \\
Residual & 68 & 526.90 & 0.49 & 1165.00 & 131.20 & 0.58 \\
Total & 116 & & & & & \\
\hline
\end{tabular}

*=Significant difference at 5\%; MLN Severity: was assessed based on CIMMYT scale (1-5) where $1=$ No MLN symptoms and $5=$ Dead heart symptoms/ complete plant death; AUDPC: area under the disease progress curve calculated from the weekly MLN severity scores based on Wilcoxson et al. (1975) equation; rAUDPC: relative Area Under Disease Progress Curve was calculated to obtain absolute values where the most MLN susceptible genotype was used for computation; \% MLN incidence: percentage of the number of leaves with MLN infection

Table 5. Weekly MLN disease progression in season 1 and MLN disease parameters

\begin{tabular}{|c|c|c|c|c|c|c|c|c|c|c|c|c|c|c|c|}
\hline \multirow[t]{2}{*}{ Entry } & \multirow[t]{2}{*}{ Genotypes } & \multicolumn{8}{|c|}{ Weekly MLN severity scores $(1-5)$} & \multirow[t]{2}{*}{ AUDPC } & \multirow[t]{2}{*}{ rAUDPC } & \multirow{2}{*}{$\begin{array}{l}\% \text { MLN } \\
\text { incidence }\end{array}$} & \multirow[t]{2}{*}{ rFS } & \multirow[t]{2}{*}{ DTS } & \multirow[t]{2}{*}{ DTP } \\
\hline & & week1 & week2 & week3 & week4 & week5 & week6 & week7 & week8 & & & & & & \\
\hline 1 & KAT25-1 & 1.67 & 2.00 & 2.33 & 3.00 & 3.33 & 3.67 & 3.67 & 4.67 & 146.00 & 90.12 & 100.00 & 4.67 & 58.08 & 64.53 \\
\hline 2 & KAT25-2 & 1.33 & 2.00 & 2.67 & 3.00 & 3.67 & 3.67 & 3.67 & 4.33 & 148.20 & 91.46 & 100.00 & 4.33 & 62.59 & 70.53 \\
\hline 3 & KAT25-3 & 0.67 & 1.67 & 2.67 & 2.33 & 3.00 & 3.00 & 3.00 & 3.00 & 120.30 & 74.28 & 100.00 & 3.00 & 59.08 & 60.33 \\
\hline 4 & KAT25-4 & 0.67 & 2.00 & 3.00 & 3.00 & 3.33 & 3.00 & 3.00 & 3.33 & 132.80 & 82.00 & 86.70 & 3.33 & 61.81 & 67.00 \\
\hline 5 & KAT25-5 & 1.00 & 1.67 & 2.33 & 2.33 & 2.67 & 2.33 & 2.33 & 3.33 & 108.80 & 67.18 & 79.20 & 3.33 & 71.77 & 70.53 \\
\hline 6 & KAT25-6 & 1.67 & 2.00 & 2.33 & 2.67 & 2.33 & 2.67 & 2.67 & 3.00 & 116.80 & 72.12 & 76.80 & 3.00 & 62.59 & 71.19 \\
\hline 7 & KAT25-7 & 1.67 & 2.00 & 2.00 & 2.00 & 2.33 & 2.00 & 2.33 & 2.67 & 101.80 & 62.86 & 66.10 & 2.67 & 63.67 & 55.67 \\
\hline 8 & KAT25-8 & 0.94 & 1.94 & 1.44 & 1.44 & 1.44 & 1.94 & 1.44 & 1.94 & 75.60 & 46.68 & 15.20 & 1.87 & 63.31 & 51.78 \\
\hline 9 & KAT25-9 & 0.67 & 1.67 & 1.33 & 1.67 & 2.33 & 2.33 & 3.00 & 2.67 & 96.50 & 59.57 & 69.40 & 2.67 & 66.77 & 64.00 \\
\hline 10 & KAT25-10 & 2.04 & 2.04 & 1.04 & 2.04 & 2.54 & 2.54 & 2.04 & 2.54 & 100.60 & 62.10 & 67.20 & 2.57 & 55.61 & 51.00 \\
\hline 11 & KAT25-11 & 1.33 & 1.67 & 2.00 & 2.67 & 2.67 & 2.33 & 2.00 & 2.33 & 104.30 & 64.40 & 44.20 & 2.33 & 70.83 & 67.33 \\
\hline 12 & KAT25-12 & 0.67 & 2.00 & 2.00 & 2.33 & 2.00 & 1.67 & 1.00 & 1.00 & 80.80 & 49.90 & 4.80 & 1.00 & 62.59 & 71.00 \\
\hline 13 & KAT25-13 & 1.33 & 1.33 & 2.00 & 2.33 & 2.00 & 2.00 & 1.33 & 1.33 & 84.70 & 52.26 & 6.70 & 1.33 & 62.59 & 71.19 \\
\hline 14 & KAT25-14 & 1.02 & 1.52 & 1.52 & 2.02 & 2.52 & 2.02 & 2.02 & 2.02 & 90.30 & 55.73 & 51.50 & 2.06 & 65.67 & 54.67 \\
\hline 15 & KAT25-15 & 1.67 & 2.33 & 2.00 & 2.67 & 2.67 & 3.00 & 3.00 & 3.00 & 123.80 & 76.44 & 93.60 & 3.00 & 59.58 & 53.03 \\
\hline 16 & KAT25-16 & 1.04 & 2.04 & 2.04 & 2.04 & 2.54 & 2.54 & 2.04 & 2.04 & 101.80 & 62.87 & 41.80 & 2.07 & 58.33 & 60.00 \\
\hline 17 & KAT25-17 & 1.33 & 2.00 & 2.67 & 2.67 & 3.00 & 3.00 & 3.00 & 3.00 & 127.20 & 78.50 & 88.90 & 3.00 & 63.31 & 53.78 \\
\hline 18 & KAT25-18 & 1.00 & 2.00 & 1.67 & 2.67 & 2.67 & 2.67 & 2.67 & 3.00 & 112.50 & 69.44 & 96.70 & 3.00 & 58.00 & 76.00 \\
\hline 19 & KAT25-19 & 1.67 & 1.33 & 1.67 & 2.00 & 2.33 & 2.00 & 1.67 & 1.67 & 87.20 & 53.81 & 17.40 & 1.67 & 63.11 & 51.69 \\
\hline 20 & KAT25-20 & 1.02 & 2.02 & 2.52 & 2.52 & 3.02 & 4.02 & 3.91 & 4.90 & 139.50 & 86.12 & 93.80 & 4.85 & 70.61 & 60.69 \\
\hline 21 & KAT25-21 & 1.67 & 2.00 & 2.00 & 2.67 & 2.67 & 2.67 & 2.67 & 2.67 & 115.80 & 71.50 & 70.40 & 2.67 & 65.81 & 71.00 \\
\hline
\end{tabular}




\begin{tabular}{|c|c|c|c|c|c|c|c|c|c|c|c|c|c|c|c|}
\hline \multirow[t]{2}{*}{ Entry } & \multirow[t]{2}{*}{ Genotypes } & \multicolumn{8}{|c|}{ Weekly MLN severity scores $(1-5)$} & \multirow[t]{2}{*}{ AUDPC } & \multirow[t]{2}{*}{ rAUDPC } & \multirow{2}{*}{$\begin{array}{l}\% \text { MLN } \\
\text { incidence }\end{array}$} & \multirow[t]{2}{*}{ rFS } & \multirow[t]{2}{*}{ DTS } & \multirow[t]{2}{*}{ DTP } \\
\hline & & week1 & week2 & week3 & week4 & week5 & week6 & week7 & week8 & & & & & & \\
\hline 22 & KAT25-22 & 1.67 & 2.00 & 3.00 & 3.00 & 3.33 & 3.33 & 3.33 & 4.33 & 144.50 & 89.20 & 100.00 & 4.33 & 62.59 & 70.60 \\
\hline 23 & KAT25-23 & 1.67 & 2.00 & 2.00 & 2.33 & 2.67 & 2.33 & 2.00 & 2.33 & 105.30 & 65.02 & 68.30 & 2.33 & 62.59 & 70.43 \\
\hline 24 & KAT25-24 & 1.00 & 2.00 & 2.00 & 2.00 & 2.00 & 2.33 & 2.33 & 2.67 & 99.50 & 61.42 & 54.20 & 2.67 & 62.59 & 71.92 \\
\hline 25 & KAT25-25 & 0.67 & 2.00 & 2.33 & 3.00 & 2.67 & 2.67 & 2.67 & 2.67 & 116.80 & 72.12 & 60.70 & 2.67 & 65.81 & 58.00 \\
\hline 26 & MLN1 & 1.02 & 2.02 & 1.52 & 1.52 & 2.02 & 2.52 & 2.02 & 2.02 & 90.00 & 55.58 & 18.90 & 2.06 & 54.85 & 60.78 \\
\hline 27 & MLN2 & 1.67 & 1.33 & 1.67 & 2.00 & 2.67 & 2.67 & 2.67 & 2.33 & 103.50 & 63.89 & 61.10 & 2.33 & 59.84 & 77.90 \\
\hline 28 & MLN3 & 1.33 & 1.67 & 2.00 & 2.00 & 2.00 & 2.00 & 2.00 & 2.33 & 90.30 & 55.76 & 56.80 & 2.33 & 71.77 & 67.28 \\
\hline 29 & MLN4 & 0.67 & 2.00 & 2.00 & 2.00 & 2.67 & 2.67 & 2.33 & 2.33 & 104.20 & 64.30 & 66.20 & 2.33 & 65.38 & 63.69 \\
\hline 30 & MLN5 & 1.00 & 1.67 & 1.00 & 1.67 & 2.33 & 2.00 & 2.00 & 3.00 & 87.30 & 53.91 & 69.10 & 3.00 & 62.59 & 67.92 \\
\hline 31 & MLN6 & 1.44 & 1.94 & 2.44 & 1.94 & 2.94 & 2.44 & 2.44 & 2.44 & 110.10 & 67.98 & 53.30 & 2.37 & 67.08 & 56.53 \\
\hline 32 & MLN7 & 1.67 & 1.67 & 1.00 & 1.33 & 2.33 & 2.33 & 2.67 & 3.00 & 94.30 & 58.23 & 100.00 & 3.00 & 54.85 & 74.91 \\
\hline 33 & MLN8 & 1.54 & 2.04 & 2.54 & 2.54 & 3.04 & 3.04 & 3.04 & 3.04 & 127.80 & 78.92 & 89.30 & 3.07 & 61.00 & 66.00 \\
\hline 34 & MLN9 & 1.33 & 1.67 & 1.00 & 1.33 & 2.00 & 2.00 & 1.67 & 1.33 & 75.70 & 46.71 & 9.10 & 1.33 & 70.83 & 64.78 \\
\hline 35 & MLN10 & 1.33 & 2.33 & 2.00 & 2.33 & 2.67 & 2.67 & 2.67 & 2.67 & 114.50 & 70.68 & 63.30 & 2.67 & 70.83 & 65.78 \\
\hline 36 & MLN11 & 0.67 & 1.67 & 2.00 & 2.33 & 3.00 & 3.33 & 3.00 & 3.00 & 118.30 & 73.05 & 77.80 & 3.00 & 59.00 & 71.33 \\
\hline 37 & MLN12 & 1.33 & 1.67 & 1.33 & 2.00 & 2.00 & 2.00 & 2.00 & 2.00 & 87.20 & 53.81 & 13.70 & 2.00 & 62.59 & 64.85 \\
\hline 38 & MLN13 & 1.33 & 2.00 & 1.67 & 2.00 & 2.33 & 2.00 & 2.00 & 2.33 & 95.00 & 58.64 & 55.50 & 2.33 & 65.84 & 70.43 \\
\hline 39 & MLN14 & 1.54 & 2.04 & 2.54 & 3.04 & 3.04 & 2.54 & 3.54 & 4.04 & 134.80 & 83.24 & 95.30 & 4.07 & 57.61 & 66.00 \\
\hline 40 & MLN15 & 1.23 & 1.82 & 1.92 & 2.24 & 2.58 & 2.56 & 2.51 & 2.66 & 107.10 & 66.13 & 63.20 & 2.66 & 59.33 & 50.00 \\
\hline 41 & MLN16 & 1.00 & 1.00 & 1.00 & 1.67 & 1.67 & 2.00 & 2.00 & 2.33 & 76.00 & 46.91 & 53.40 & 2.33 & 56.61 & 63.69 \\
\hline 42 & MLN17 & 1.33 & 1.33 & 1.67 & 1.67 & 2.00 & 2.33 & 2.33 & 2.33 & 90.70 & 55.97 & 52.40 & 2.33 & 54.85 & 72.78 \\
\hline 43 & MLN18 & 0.67 & 1.33 & 1.33 & 2.00 & 2.33 & 2.33 & 2.33 & 2.33 & 90.80 & 56.07 & 50.40 & 2.33 & 55.79 & 74.28 \\
\hline 44 & MLN19 & 1.33 & 1.33 & 1.67 & 1.33 & 2.33 & 2.67 & 2.33 & 2.00 & 91.80 & 56.69 & 51.10 & 2.00 & 62.59 & 69.19 \\
\hline 45 & MLN20 & 1.00 & 2.33 & 1.33 & 2.00 & 2.00 & 2.33 & 2.00 & 2.00 & 92.70 & 57.20 & 31.20 & 2.00 & 59.39 & 70.33 \\
\hline 46 & MLN21 & 1.00 & 2.00 & 2.00 & 1.67 & 3.33 & 2.67 & 3.00 & 3.00 & 114.70 & 70.78 & 62.10 & 3.00 & 64.58 & 66.33 \\
\hline 47 & MLN22 & 1.04 & 2.04 & 1.54 & 2.54 & 2.54 & 2.54 & 2.04 & 2.04 & 102.10 & 63.02 & 48.00 & 2.07 & 60.00 & 67.00 \\
\hline 48 & MLN23 & 1.67 & 2.00 & 2.00 & 2.67 & 3.00 & 3.00 & 3.00 & 3.00 & 124.00 & 76.54 & 89.80 & 3.00 & 55.79 & 73.60 \\
\hline 49 & MLN24 & 1.33 & 2.00 & 2.33 & 2.00 & 2.33 & 2.67 & 2.67 & 2.67 & 109.80 & 67.80 & 62.80 & 2.67 & 70.37 & 68.53 \\
\hline 50 & MLN25 & 1.00 & 2.00 & 2.00 & 2.67 & 3.00 & 3.00 & 3.00 & 3.00 & 121.70 & 75.10 & 70.10 & 3.00 & 70.61 & 67.67 \\
\hline 51 & MLN26 & 1.33 & 1.67 & 1.67 & 2.33 & 2.33 & 2.33 & 2.00 & 2.00 & 96.30 & 59.47 & 38.50 & 2.00 & 56.78 & 69.78 \\
\hline 52 & MLN27 & 1.33 & 1.33 & 1.00 & 1.33 & 2.00 & 2.00 & 2.33 & 2.67 & 82.80 & 51.13 & 67.90 & 2.67 & 62.59 & 70.43 \\
\hline 53 & MLN28 & 1.00 & 1.67 & 1.33 & 2.00 & 2.33 & 2.33 & 2.67 & 3.00 & 98.80 & 61.01 & 83.60 & 3.00 & 55.31 & 74.00 \\
\hline 54 & MLN29 & 2.00 & 2.00 & 1.33 & 2.00 & 2.67 & 2.67 & 2.67 & 2.33 & 106.80 & 65.95 & 41.20 & 2.33 & 62.84 & 67.92 \\
\hline 55 & MLN30 & 1.67 & 1.67 & 1.33 & 2.00 & 2.33 & 2.00 & 1.67 & 1.67 & 87.20 & 53.81 & 23.90 & 1.67 & 70.37 & 63.45 \\
\hline 56 & MLR-1 & 1.00 & 2.00 & 2.33 & 2.33 & 2.67 & 3.04 & 2.54 & 3.04 & 119.10 & 73.52 & 74.90 & 3.07 & 63.78 & 72.28 \\
\hline 57 & MLR-2 & 1.00 & 1.67 & 1.00 & 1.33 & 1.67 & 2.00 & 2.00 & 2.00 & 76.80 & 47.43 & 45.40 & 2.00 & 70.61 & 68.33 \\
\hline 58 & MLR-3 & 2.04 & 2.04 & 3.04 & 3.04 & 3.04 & 2.54 & 3.54 & 4.04 & 139.80 & 86.32 & 93.60 & 4.07 & 59.39 & 63.69 \\
\hline 59 & MLR-4 & 2.00 & 2.00 & 2.67 & 3.33 & 3.33 & 3.00 & 3.33 & 3.00 & 138.80 & 85.70 & 90.60 & 3.00 & 70.83 & 65.69 \\
\hline 60 & MLR-5 & 1.94 & 1.44 & 2.44 & 2.94 & 2.94 & 2.94 & 2.94 & 2.94 & 124.40 & 76.77 & 76.70 & 2.87 & 59.00 & 54.67 \\
\hline 61 & MLR-6 & 1.00 & 1.67 & 2.33 & 2.67 & 3.00 & 2.67 & 2.67 & 2.67 & 115.80 & 71.50 & 73.60 & 2.67 & 60.78 & 61.53 \\
\hline 62 & MLR-7 & 0.44 & 1.94 & 1.44 & 1.44 & 2.44 & 2.44 & 2.44 & 2.94 & 94.90 & 58.56 & 68.90 & 2.87 & 64.81 & 62.67 \\
\hline 63 & MLR-8 & 1.33 & 1.67 & 2.33 & 2.67 & 2.67 & 2.67 & 2.67 & 2.33 & 113.50 & 70.06 & 56.40 & 2.33 & 60.00 & 70.33 \\
\hline 64 & MLR-9 & 1.00 & 2.00 & 2.00 & 2.67 & 2.67 & 3.00 & 3.00 & 3.00 & 119.30 & 73.66 & 85.60 & 3.00 & 64.58 & 56.53 \\
\hline 65 & MLR-10 & 0.92 & 1.92 & 1.92 & 1.92 & 2.91 & 2.91 & 2.91 & 2.91 & 112.30 & 69.31 & 74.00 & 2.86 & 60.31 & 50.28 \\
\hline 66 & MLR-11 & 0.67 & 1.33 & 1.33 & 2.00 & 2.00 & 2.00 & 2.00 & 2.00 & 82.70 & 51.03 & 29.70 & 2.00 & 68.11 & 63.67 \\
\hline 67 & MLR-12 & 1.54 & 1.54 & 1.54 & 2.04 & 2.54 & 2.54 & 2.04 & 2.54 & 98.80 & 61.02 & 58.50 & 2.57 & 58.00 & 68.00 \\
\hline 68 & MLR-13 & 0.67 & 2.00 & 1.67 & 2.00 & 2.33 & 2.33 & 2.33 & 2.33 & 97.30 & 60.08 & 53.90 & 2.33 & 59.39 & 61.53 \\
\hline 69 & MLR-14 & 0.67 & 1.67 & 2.00 & 2.67 & 3.00 & 3.00 & 3.33 & 3.33 & 121.80 & 75.21 & 93.90 & 3.33 & 62.59 & 70.60 \\
\hline 70 & MLR-15 & 0.67 & 1.67 & 2.33 & 2.00 & 2.67 & 2.67 & 3.00 & 2.67 & 110.00 & 67.90 & 75.40 & 2.67 & 59.00 & 62.33 \\
\hline 71 & MLR-16 & 1.33 & 1.67 & 2.33 & 3.00 & 3.00 & 2.67 & 2.67 & 2.67 & 119.30 & 73.66 & 75.00 & 2.67 & 62.08 & 56.03 \\
\hline 72 & MLR-17 & 1.33 & 2.00 & 2.33 & 2.67 & 2.67 & 2.67 & 2.67 & 2.00 & 116.80 & 72.12 & 63.60 & 2.00 & 62.59 & 60.94 \\
\hline 73 & check & 1.33 & 2.33 & 2.00 & 2.33 & 2.67 & 2.67 & 2.33 & 2.33 & 111.00 & 68.52 & 50.00 & 2.33 & 62.33 & 50.00 \\
\hline \multicolumn{10}{|c|}{ Least significant difference } & 31.5 & 19.5 & 47.0 & 1.2 & 8.19 & 14.65 \\
\hline \multicolumn{10}{|c|}{$\%$ Coefficient of variation } & 5.3 & 5.3 & 12.4 & 8.8 & 1.10 & 1.30 \\
\hline
\end{tabular}

*=Significant difference at 5\%; MLN Severity: was assessed based CIMMYT scale (1-5) where $1=$ No MLN symptoms and $5=$ Dead heart symptoms/ complete plant death; AUDPC: area under the disease progress curve calculated from the weekly MLN severity scores based on Wilcoxson et al. (1975) equation; rAUDPC: relative Area Under Disease Progress Curve calculated as a percentage of the AUDPC; \% MLN incidence: percentage of the number of leaves with MLND infection; DTS= Days to 50\% silking, DTP= Days to 50\% Pollen shed 
Table 6. Weekly MLN disease progression in season 2 and other MN disease parameters

\begin{tabular}{|c|c|c|c|c|c|c|c|c|c|c|c|c|c|c|c|}
\hline \multirow[t]{2}{*}{ Entry } & \multirow[t]{2}{*}{ Genotype } & \multicolumn{7}{|c|}{ Weekly MLN severity scores $(1-5)$} & \multirow[t]{2}{*}{ AUDPC } & \multirow[t]{2}{*}{ rAUDPC } & \multirow[t]{2}{*}{ FS } & \multirow[t]{2}{*}{ rFS } & \multirow{2}{*}{$\begin{array}{l}\% \text { MLN } \\
\text { incidence }\end{array}$} & \multirow[t]{2}{*}{ DTP } & \multirow[t]{2}{*}{ DTS } \\
\hline & & week1 & week2 & week3 & week4 & week5 & week6 & week7 & & & & & & & \\
\hline 1 & MLN1 & 1.09 & 1.09 & 2.09 & 2.09 & 2.09 & 2.09 & 4 & 143 & 48 & 4 & 4 & 43 & 93.5 & 89.1 \\
\hline 2 & MLN2 & 1.92 & 1.92 & 2.92 & 2.92 & 2.92 & 2.92 & 4 & 187 & 63 & 4 & 4 & 97 & 85.3 & 78.6 \\
\hline 3 & MLN3 & 2.00 & 2.00 & 2.00 & 2.00 & 2.33 & 3.00 & 4 & 172 & 58 & 4 & 4 & 98 & 92.7 & 87.3 \\
\hline 4 & MLN4 & 2.00 & 2.00 & 2.00 & 2.00 & 2.00 & 3.00 & 4 & 167 & 56 & 4 & 4 & 81 & 90 & 83 \\
\hline 5 & MLN5 & 2.00 & 2.00 & 2.00 & 2.00 & 2.00 & 3.00 & 4 & 176 & 59 & 4 & 4 & 97 & 89.5 & 90 \\
\hline 6 & MLN6 & 1.09 & 1.09 & 1.09 & 1.09 & 1.09 & 2.59 & 3 & 130 & 44 & 3 & 3 & 63 & 84 & 80.6 \\
\hline 7 & MLN7 & 1.92 & 2.92 & 3.42 & 3.42 & 3.42 & 4.42 & 4 & 241 & 81 & 4 & 4 & 97 & 85.8 & 97.3 \\
\hline 8 & MLN8 & 1.16 & 2.16 & 2.16 & 2.16 & 3.16 & 3.16 & 3 & 179 & 60 & 3 & 3 & 83 & 85.8 & 80.9 \\
\hline 9 & MLN9 & 1.33 & 1.33 & 1.33 & 1.33 & 1.67 & 2.67 & 3 & 135 & 45 & 3 & 3 & 55 & 88.7 & 83 \\
\hline 10 & MLN10 & 1.59 & 2.09 & 2.09 & 2.09 & 2.09 & 3.09 & 3 & 162 & 54 & 3 & 3 & 69 & 85.4 & 85.6 \\
\hline 11 & MLN11 & 2.33 & 3.00 & 3.00 & 3.00 & 3.33 & 3.67 & 4 & 217 & 73 & 4 & 4 & 100 & 84 & 84.3 \\
\hline 12 & MLN12 & 1.01 & 1.01 & 1.01 & 1.01 & 2.01 & 2.01 & 3 & 123 & 41 & 3 & 3 & 26 & 93.7 & 94.3 \\
\hline 13 & MLN13 & 2.09 & 2.09 & 2.09 & 2.09 & 3.09 & 3.59 & 4 & 193 & 65 & 4 & 4 & 100 & 87.5 & 85.1 \\
\hline 14 & MLN14 & 1.66 & 1.91 & 2.04 & 2.04 & 2.27 & 2.94 & 3 & 165 & 56 & 3 & 3 & 74 & 85.8 & 80.9 \\
\hline 15 & MLN15 & 1.50 & 1.50 & 1.50 & 1.50 & 2.00 & 2.50 & 3 & 147 & 49 & 4 & 4 & 47 & 92.1 & 87.8 \\
\hline 16 & MLN16 & 3.16 & 3.16 & 3.16 & 3.16 & 3.16 & 4.16 & 4 & 239 & 80 & 4 & 4 & 74 & 85.8 & 91.9 \\
\hline 17 & MLN17 & 1.50 & 1.50 & 1.50 & 1.50 & 1.50 & 2.50 & 2 & 126 & 42 & 3 & 3 & 27 & 92.6 & 89.8 \\
\hline 18 & MLN18 & 0.92 & 1.42 & 1.42 & 1.42 & 1.42 & 2.42 & 3 & 126 & 42 & 3 & 3 & 34 & 92.3 & 89.1 \\
\hline 19 & MLN19 & 1.33 & 1.33 & 1.67 & 1.67 & 1.67 & 2.33 & 3 & 127 & 43 & 3 & 3 & 38 & 95 & 92.7 \\
\hline 20 & MLN20 & 1.92 & 1.92 & 1.92 & 1.92 & 2.42 & 3.42 & 3 & 175 & 59 & 3 & 3 & 97 & 88.3 & 89.1 \\
\hline 21 & MLN21 & 2.00 & 2.33 & 2.33 & 2.33 & 2.67 & 3.33 & 4 & 191 & 64 & 4 & 4 & 75 & 86 & 82 \\
\hline 22 & MLN22 & 1.01 & 1.01 & 2.01 & 2.01 & 2.01 & 3.01 & 4 & 162 & 54 & 4 & 4 & 80 & 93.7 & 90.3 \\
\hline 23 & MLN23 & 1.66 & 1.91 & 2.04 & 2.04 & 2.27 & 2.94 & 3 & 165 & 56 & 3 & 3 & 74 & 85.8 & 80.9 \\
\hline 24 & MLN24 & 1.59 & 2.09 & 2.09 & 2.09 & 2.09 & 2.59 & 3 & 153 & 51 & 3 & 3 & 66 & 93.3 & 83.6 \\
\hline 25 & MLN25 & 1.33 & 1.67 & 2.00 & 2.00 & 2.33 & 2.67 & 3 & 147 & 49 & 3 & 3 & 61 & 88.7 & 82 \\
\hline 26 & MLN26 & 1.33 & 2.33 & 2.33 & 2.33 & 2.67 & 3.00 & 3 & 169 & 57 & 3 & 3 & 79 & 86.8 & 81.7 \\
\hline 27 & MLN27 & 1.67 & 2.00 & 2.00 & 2.00 & 2.00 & 2.67 & 3 & 151 & 51 & 3 & 3 & 69 & 90 & 84 \\
\hline 28 & MLN28 & 1.50 & 1.50 & 1.50 & 1.50 & 2.00 & 2.00 & 3 & 131 & 44 & 3 & 3 & 56 & 90.9 & 87.8 \\
\hline 29 & MLN29 & 1.00 & 1.33 & 1.33 & 1.33 & 1.33 & 2.00 & 2 & 109 & 36 & 2 & 2 & 51 & 88.7 & 85.3 \\
\hline 30 & MLN30 & 1.33 & 2.00 & 2.00 & 2.00 & 2.00 & 3.00 & 3 & 158 & 53 & 3 & 3 & 94 & 90.3 & 84 \\
\hline 31 & MLR1 & 1.33 & 1.33 & 1.67 & 1.67 & 1.67 & 2.33 & 3 & 137 & 46 & 3 & 3 & 44 & 86.7 & 82.7 \\
\hline 32 & MLR2 & 1.59 & 1.59 & 1.59 & 1.59 & 2.09 & 3.59 & 5 & 188 & 63 & 5 & 5 & 93 & 89.7 & 82.1 \\
\hline 33 & MLR3 & 1.33 & 1.33 & 2.00 & 2.00 & 2.00 & 3.00 & 4 & 160 & 54 & 4 & 4 & 92 & 77.3 & 67.7 \\
\hline 34 & MLR4 & 1.33 & 2.00 & 2.00 & 2.00 & 2.00 & 2.33 & 3 & 141 & 47 & 3 & 3 & 29 & 90.1 & 82.8 \\
\hline 35 & MLR5 & 2.00 & 3.33 & 3.33 & 3.33 & 4.00 & 4.33 & 4 & 246 & 83 & 4 & 4 & 100 & 81.1 & 72.3 \\
\hline 36 & MLR6 & 1.67 & 1.67 & 1.67 & 1.67 & 2.00 & 3.00 & 3 & 154 & 52 & 3 & 3 & 97 & 74.7 & 70 \\
\hline 37 & MLR7 & 2.00 & 2.33 & 2.67 & 2.67 & 3.00 & 3.33 & 4 & 194 & 65 & 4 & 4 & 84 & 88.7 & 80.7 \\
\hline 38 & MLR8 & 1.33 & 1.67 & 1.67 & 1.67 & 2.00 & 2.33 & 3 & 144 & 48 & 3 & 3 & 67 & 83 & 79.7 \\
\hline 39 & MLR9 & 1.33 & 1.33 & 1.33 & 1.33 & 2.00 & 2.67 & 3 & 145 & 49 & 3 & 3 & 88 & 77.3 & 66.3 \\
\hline 40 & MLR10 & 2.00 & 2.00 & 2.00 & 2.00 & 2.00 & 3.00 & 4 & 178 & 60 & 5 & 5 & 94 & 87.1 & 79.3 \\
\hline 41 & MLR11 & 2.09 & 2.59 & 2.59 & 2.59 & 2.59 & 3.09 & 4 & 185 & 62 & 4 & 4 & 90 & 79 & 71.1 \\
\hline 42 & MLR12 & 2.00 & 2.00 & 2.33 & 2.33 & 2.33 & 2.67 & 3 & 161 & 54 & 3 & 3 & 58 & 83.3 & 73.3 \\
\hline 43 & MLR13 & 1.33 & 1.33 & 1.67 & 1.67 & 2.00 & 2.67 & 3 & 147 & 49 & 3 & 3 & 75 & 78.7 & 72 \\
\hline 44 & MLR14 & 2.00 & 2.33 & 2.33 & 2.33 & 2.67 & 3.67 & 4 & 193 & 65 & 4 & 4 & 89 & 73.5 & 67.6 \\
\hline 45 & MLR15 & 3.00 & 3.33 & 3.33 & 3.33 & 3.33 & 4.00 & 4 & 235 & 79 & 4 & 4 & 94 & 80.7 & 73.3 \\
\hline 46 & MLR16 & 1.67 & 2.00 & 2.00 & 2.00 & 2.00 & 3.00 & 3 & 161 & 54 & 3 & 3 & 97 & 77.3 & 67.3 \\
\hline 47 & MLR17 & 2.00 & 2.00 & 2.00 & 2.00 & 2.00 & 2.50 & 2 & 141 & 47 & 3 & 3 & 70 & 81.6 & 70.3 \\
\hline 48 & MLR18 & 1.33 & 1.67 & 1.67 & 1.67 & 2.00 & 2.67 & 3 & 149 & 50 & 3 & 3 & 75 & 71.3 & 66.3 \\
\hline \multicolumn{9}{|c|}{ Least significant difference } & 21.02 & 7.05 & & 0.47 & 14.14 & 6.62 & 8.623 \\
\hline \multicolumn{9}{|c|}{$\%$ Coefficient of variation } & 6.90 & 6.90 & & 6.50 & 11.30 & 1 & 2.4 \\
\hline
\end{tabular}

*=Significant difference at 5\%; MLN Severity: was assessed based CIMMYT scale (0-1) where $0=$ No MLN symptoms and $5=$ Dead heart symptoms/ complete plant death; AUDPC: area under the disease progress curve calculated from the weekly MLN severity scores based on Wilcoxson et al., (1975) equation; ; rAUDPC: relative Area Under Disease Progress Curve calculated as a percentage of the AUDPC; \% MLN incidence: percentage of the number of leaves with MLND infection; LSD=Least significant difference, $\mathrm{CV}=\mathrm{Coefficient}$ of variation DTS= Days to $50 \%$ silking, DTP= Days to $50 \%$ Pollen shed 
Table 7. Correlation among the traits across two seasons

\begin{tabular}{|c|c|c|c|c|c|c|c|c|c|c|c|c|}
\hline Trait & AUDPC & DTP & DTS & FS & $\%$ DI & week1 & week2 & week3 & week4 & week5 & week6 & Week7 \\
\hline AUDPC & - & & & & & & & & & & & \\
\hline DTP & $0.43^{*}$ & - & & & & & & & & & & \\
\hline DTS & $0.47 *$ & $0.90 *$ & - & & & & & & & & & \\
\hline FS & $0.85^{*}$ & $0.31 *$ & $0.35^{*}$ & - & & & & & & & & \\
\hline$\% \mathrm{DI}$ & $0.64 *$ & 0.11 & $0.16^{*}$ & $0.64 *$ & - & & & & & & & \\
\hline week1 & $0.51^{*}$ & -0.09 & -0.02 & $0.40^{*}$ & $0.42 *$ & - & & & & & & \\
\hline week2 & $0.57 *$ & -0.11 & -0.07 & $0.40^{*}$ & $0.38^{*}$ & 0.73* & - & & & & & \\
\hline week3 & $0.54 *$ & $-0.23^{*}$ & $-0.20 *$ & $0.43^{*}$ & $0.40^{*}$ & $0.64 *$ & $0.84 *$ & - & & & & \\
\hline week4 & $0.59 *$ & $-0.16^{*}$ & $-0.14^{*}$ & $0.47 *$ & $0.45^{*}$ & $0.60 *$ & $0.77^{*}$ & $0.90 *$ & - & & & \\
\hline week5 & $0.66 *$ & -0.09 & -0.04 & $0.53 *$ & $0.48^{*}$ & 0.61* & 0.77* & $0.88 *$ & $0.92 *$ & - & & \\
\hline week6 & $0.82 *$ & 0.20 * & $0.22 *$ & $0.69 *$ & $0.60 *$ & $0.57 *$ & 0.61* & 0.64* & 0.72* & $0.75^{*}$ & - & \\
\hline week7 & $0.84 *$ & $0.36^{*}$ & $0.36^{*}$ & $0.86^{*}$ & $0.56^{*}$ & $0.44 *$ & $0.45^{*}$ & $0.46^{*}$ & $0.54 *$ & $0.60 *$ & $0.81 *$ & - \\
\hline
\end{tabular}

*=Significant difference at 5\%; MLN Severity: was assessed based CIMMYT scale (0-1) where $0=$ No MLN symptoms and $5=$ Dead heart symptoms/ complete plant death; AUDPC: area under the disease progress curve calculated from the weekly MLN severity scores based on Wilcoxson et al. (1975) equation; \% MLN incidence: percentage of the number of leaves with MLN infection; DTS= Days to 50\% silking, DTP= Days to $50 \%$ Pollen shed

\section{Discussion and Conclusion}

Continuous efforts to identify resistant sources of MLN for introgression into the adapted maize genotypes are imperative in combating the sporadic nature of MLN causal agents especially in the face of climate change. The current research which involved assessing the response of assorted maize genotypes to the MLN disease has revealed the reaction of the genetically divergent maize genotypes. The plants exhibited clear mosaic symptoms on leaves, systemic in nature and which were indicative of susceptibility to MLN. Significant differences were reported among the genotypes with response to the different MLN assessment parameters. When a virus infects a plant, a signal transduction leads to physiological changes in the host plant implying that the genetic structure of the plants play a critical role in the infection and spread of viruses (Salaudeen and Aguguom, 2014). The significant and positive correlation coefficients noted between the MLN disease parameters validated their use in the assessment of the genotypes for response to MLN. Previous studies have identified susceptible and resistant genotypes by using the same parameters (Zambrano et al., 2013).

More than 50\% of the genotypes screened in the two seasons were susceptible to the disease, validating the risk that MLN poses to maize production in Kenya and its food security status. These findings corroborate with reports by Gowda et al. (2015) on the high susceptibility of Kenyan maize germplasm to the MLN especially under artificial MLN infections. The MLN infection was induced by the artificial inoculation of SCMV and MCMV suggesting that the two viruses increased the viral load leading to the symptomatic effects which are both additive and lethal (Marçon et al., 1997). This study involved CIMMYT germplasm previously depicted as resistant in other studies. In this study, some lines still exhibited resistant responses namely MLN1, MLN15, MLN16, MLN17, MLN19, MLN18 and MLN12 which had low MLN severity ranging from 2-3 and also statistically low AUDPC, rAUDPC and \% MLN incidence values. However, most of the other CIMMYT lines succumbed to the MLN disease.

Clear symptoms were observed among the susceptible MLN lines. Symptoms arise from interactions of the host with the virus and are as a result of compatible interaction which lead to the diversion of assimilates from the host plant to favour the virus cellular processes like its replication and multiplication (Revers et al., 1999). The resistance on the other hand could imply an incompatible interaction which could have been stimulated by the rapid necrosis at the foci of virus entry preventing its further spread and this could probably explain the partial resistance observed among some of the maize genotypes in this research work (Ronde et al., 2014). Previous studies have reported intense susceptibility to MLN in East Africa (EA) among the pre-commercial and commercial maize varieties (Jumbo et al., 2015; Semagn et al., 2015). It can be deduced that the use of germplasm labelled as resistant or moderately resistant should be done with caution and there is need for validation through repeat experiments over several seasons with reliable inoculations (CIMMYT, 2013; Gowda et al., 2015).

Among the farmer varieties, MLR-10, MLR-13 and MLR-17 MLR4, MLR6, MLR9, MLR18 and MLR16 were superior for MLN resistance These genotypes combined low AUDPC values which implies that the plant defense mechanism against the viruses could be mediated by resistance $(\mathrm{R})$ genes which are observed as complete resistance or extreme resistance (ER) and that the virus replication could have been hindered or gone 
undetectable among the infected cells (Ingvardsen et al., 2010). The farmer varieties are highly heterogeneous due to out-crossing within the farmers' fields. The presence of some resistance among these genotypes could offer the much-needed alternative to resource-constrained Kenyan farmers in combating the MLN threat.

This study also revealed that none of the genotypes was immune to the MLN disease across the two seasons of evaluation. However, some genotypes showed clear hypersensitive responses and necrotic symptoms. Previous studies by Zambrano et al. (2014) have reported presence of some resistance to multiple viruses in the family potyviridae. The presence of passive and active defense mechanisms hinders virus multiplication and spread influencing either the susceptibility or the resistance of germplasm (Zambrano et al., 2014).

The weekly MLN progression among the maize genotypes gave a great insight into the virus dynamics. Among the most susceptible genotypes, there was high AUDPC values coupled with high MLN scores as the weeks progressed. Revers et al. (1999) explained that the Potyviruses tend to follow the sink to source criteria in photo-assimilate partitioning. The plants viruses are known to gain access to the plant followed by the viral interaction with the host cells. This leads to the manipulation of the host cell pathways into viral factories (Sharma and Misra, 2011). Further on, the systemic infection of the viruses from the primary infection point and its invasion of the distal regions through the mesophyll into the bundle sheath cells, phloem parenchyma, and companion cells into phloem sieve elements is through passive translocation in the phloem leading to further infection and spread (Revers et al., 1999). However, among some genotypes, the symptoms plateaued while in others there was increased MLN severity with time. The MLN causal viruses cause systemic infections and the virus translocation from the point of inoculation depends on the cell-to-cell movement of its particles after the viral replication and establishment (Salaudeen and Aguguom, 2014). The resistant genotypes with low AUDPC values on the other hand could have had the inherent ability to retard the virus infection by inhibiting the movement of the virus inside the host cells (Gowda et al, 2015).

The superior maize genotypes identified in this research could serve as potential donors to improve the adapted maize varieties to combat the MLN threat in Kenya. However, the low frequency of resistance sources is an issue of great concern and this affects the nutritional and food security status of Kenya which is highly dependent on maize as a key staple food crop. Further evaluation of these elite genotypes for response to the singular viruses and establishment of the genetics of the MLN resistance will enhance their efficient utilization in breeding programs. Through genetic studies, the nature of the genes conditioning resistance to the MLN and its causal virus will help to elucidate further the viruses' dynamics.

\section{Acknowledgements}

This work was jointly supported by the Alliance for Green Revolution in Africa (AGRA) and the Association for Strengthening Agricultural Research in Eastern and Central Africa (ASARECA). The authors acknowledge the immense support from KALRO and CIMMYT for the provision of the maize germplasm.

\section{References}

Adams, I. P., Miano, D.W., Kinyua, M. Z., Wangai, A., Kimani, E., Phiri, N., ... Boonham, N. (2013). Use of next-generation sequencing for the identification and characterization of Maize chlorotic mottle virus and Sugarcane mosaic virus causing maize lethal necrosis in Kenya. Plant Pathology. 62, 741-749. https://doi.org/10.1111/j.1365-3059.2012.02690.x

Cabanas, D., Watanabe, S., Higashi, C. H. V., \& Bressan, A. (2013). Dissecting the Mode of Maize Chlorotic Mottle Virus Transmission (Tombusviridae: Machlomovirus) by Frankliniella williamsi (Thysanoptera: Thripidae). J Econ Entomol, 106, 16-24. https://doi.org/10.1603/EC12056

CABI., (2015). Invasive species compendium, Datasheets, Maps, Images, Abstracts and full-text invasive species of the world. http://www.cabi.org/isc/

CIMMYT. (2013). Kiboko Crops Research Station: A brief and visitors' guide. CIMMYT.

FSNWG. (2012). Regional Food Security Situation and Outlook. Food Security and Nutrition Working Group, http://www. disasterriskreduction.net/east-central-africa/ fsnwg.

Gowda, M., Das, B., Makumbi, D., Babu, R., Kassa Semagn, K., Mahuku, G., Olsen, M.S., Bright, M. J., Beyene, Y., \& Prasanna, M. B. (2015). Genome-wide association and genomic prediction of resistance to maize lethal necrosis disease in tropical maize germplasm.TheorAppl Genet, 127, 867-880. https://doi.org/10.1007/s00122-015-2559-0

Ingvardsen, C. R., Xing,Y., Frei, U. K., \& Lübberstedt, T. (2010). Genetic and physical Wne mapping of Scmv2, a potyvirus resistance gene in maize. Theor Appl Genet, 120, 1621-1634. 
https://doi.org/10.1007/s00122-010-1281-1

Jones, M. W., Boyd, E. C., \& Redinbaugh, M. G. (2011). Responses of maize (Zea mays L.) near isogenic lines carrying Wsm1, Wsm2, and Wsm3 to three viruses in the Potyviridae. Theor Appl Genet, 123, 729-740. https://doi.org/10.1007/s00122-011-1622-8

Jumbo, M. B., Makumbi, D, Kimunye, J. N., Mahuku, G., Bekunda, M., \& Hoeschle-Zeledon, I. (2015). Integration of maize lethal necrosis disease management in crop-livestock intensification to enehance productivity of smallholder agricultural production systems in East Africa-An Africa RISING approach. Poster prepared for the International conference on Integrated Systems Research, Ibadan, Nigeria, 3-6 March 2015. Nairobi, Kenya: CIMMYT.

Karyeija, R. F., Kreuze, J. F., Gibson, W. R., \& Valkonen, J. P. T. (2000). Synergistic Interactions of a Potyvirus and a Phloem-Limited Crinivirus in Sweet Potato Plants. Virology, 269, 26-36. https://doi.org/10.1006/viro.1999.0169

Keya, S., \& Rubaihayo, P. (2013). Progresson on-farm production and productivity in East Africa Community; 50 years after independence. Kilimo trust technical paper No.8. International symposium on agriculture, EAC partner states at 50 years. 5-7 November, 2013.

Kiruwa, H. F., Feyissa, T., \& Ndakidemi, A. P. (2016). Insight of maize lethal necrotic disease. A major constraint to maize production in East Africa: African journal of microbiology Research, 10(9), 271-279. https://doi.org/10.5897/AJMR2015.7534

Marçon, A., Kaeppler, S. M., \& Jensen, S. G. (1997). Genetic variability among maize inbred lines for resistance to the high plains virus-wheat streak mosaic virus complex. Plant Dis., 81, 195-198. https://doi.org/10.1094/PDIS.1997.81.2.195

Mbega, E. R., Ndakidemi, P. A., Mamiro, D. P., Mushongi, A. A., Kitenge, K. M., \& Ndomba, O. A. (2016). Role of potyviruses in synergistic interaction leading to maize lethal necrotic disease on maize. International journal of current microbiology and applied sciences, 5(6), 85-96. https://doi.org/10.20546/ijcmas.2016.506.011

Melchinger, A. E., Kuntze, L.,Gumber, R. K., Lu bberstedt, T., \& Fuchs, E. (1998). Genetic basis of resistance to sugarcane mosaic virus in European maize germplasm. Theor Appl Genet, 96, 1151-1161. https://doi.org/10.1007/s001220050851

Miano, F., Kabaki, J., \& Viollet, D. (2013). Controlling Maize Lethal Necrosis Disease via Vector Management. AFSTA

Nelson, S., Brewbaker, J., \& Hu, J. (2011). Maize Chlorotic Mottle Virus. Plant Disease, 79, 1-6.

Olsen, M., Yao, N., Tadesse, B., Das, B., Gowda, M., Semagn, K., Jumbo, M., \& Killian, A. (2016). Mapping genetic regions associated with MLND using QTL-Seq. Biosciences eastern and central Africa.

Onyango, C. M., Harbinson, J., Imungi, J. K., Onwonga, R. N., \& Kooten, O. (2012). Effect of Nitrogen Source, Crop Maturity Stage, and Storage Conditions on Phenolics and Oxalate Contents in Vegetable Amaranth (Amaranthus hypochondriacus). Journal of Agricultural Science, 4(7), 219-230. https://doi.org/10.5539/jas.v4n7p219

Orawu, M., Melis R., Laing M., \& Derera J. (2013). Genetic inheritance of resistance to cowpea aphid-borne mosaic virus in cowpea. Euphytica, 189, 191-201. https://doi.org/10.1007/s10681-012-0756-3

Redinbaugh, M. G., Seifers, D. L., Meulia, T., Abt, J. J., Anderson, R. J., Styer, W. E., Ackerman, J., Salomon, R., Houghton, W., Creamer, R.,Gordon, D. T., \& Hogenhout, S. A. (2002). Maize fine streak virus, a new leafhopper-transmitted rhabdovirus. Phytopathology, 92, 1167-1174. https://doi.org/10.1094/PHYTO.2002.92.11.1167

Redinbaugh, M. G., Jones, M. W., \& Gingery, R. E. (2004). The genetics of virus resistance in maize (Zea mays L.). Maydica, 49, 187-190.

Revers, F., Le Gall, O., Candresse, T., \& Maule, A. J. (1999). New Advances in Understanding the Molecular Biology of Plant/Potyvirus Interactions. MPMI, 12(5), 367-376. https://doi.org/10.1094/MPMI.1999.12.5.367

Ronde, D., Butterbach, P., \& Kormelink, R. (2014). Dominant resistance against plant viruses.

Salaudeen, M. T., \& Aguguom, A. (2014). Identification of some cowpea accessions tolerant to cowpea mild 
mottle virus. I.J.S.N. 5(2), 261-267.

Semagn, K., Beyene, Y., Babu, R., Nair S., Gowda, M., Das, B., Tarekegne A., Mugo S., Mahuku, G., Worku M., Warburton L. M., Olsen, M., \& Prassana, B. M. (2015). Quantitative Trait Loci Mapping and molecular breeding for Developing stress resilient maize for Sub-Saharan Africa. Crop Sci., 55, 1-11. https://doi.org/10.2135/cropsci2014.09.0646

Sharma, K., \& Misra, R. S. (2011). Molecular approaches towards analyzing the viruses infecting maize (Zea mays L.). Journal of General and Molecular Virology, 3(1), 1-17.

Shi, X. M., Miller, H., Verchot, J., Carrington, J. C., \& Vance, V. B. (1997). Mutations in the Region Encoding the Central Domain of Helper Component-Proteinase (HC-Pro) Eliminate Potato Virus X/Potyviral Synergism. Virology, 231, 35-42. https://doi.org/10.1006/viro.1997.8488

Wangai, A. W., Redinbaugh, M. G., Kinyua, Z. M., Miano, D. W., Leley, P. K., Kasina, M., Mahuku, G., Scheets, K., \& Jeffers. (2012). First Report of Maize chlorotic mottle virus and Maize Lethal Necrosis in Kenya. Plant Disease, 96, 1582. https://doi.org/10.1094/PDIS-06-12-0576-PDN

Wilcoxson, R. D., Skovmand, B., \& Atif, A. H. (1975). Evaluation of wheat cultivars for ability to retard development of stem rust. Annual Applied Biology, 80, 275-281. https://doi.org/10.1111/j.1744-7348.1975.tb01633.x

Wu, L., Wang, S., Chen, X., Wang, X., Wu, L., \& Zu, X. (2013). Proteomic and Phytohormone Analysis of the Response of Maize (Zea mays L.) Seedlings to Sugarcane Mosaic Virus. PLoS ONE, 8(7), e70295. https://doi.org/10.1371/journal.pone.0070295

Xu, M. L., Melchinger, A. E., \& Lübberstedt, T. (2000). Origin of Scm1 and Scm2 - two loci conferring resistance to sugarcane mosaic virus (SCMV) in maize. Theor Appl Genet, 100, 934-94. https://doi.org/10.1007/s001220051373

Yang, Z. N., \& Mirkov, T. E. (1997). Sequence and relationships of sugarcane mosaic and sorghum mosaic virus strains and development of RTPCR-based RFLPs for strain discrimination. Phytopathology, 87, 932-939. https://doi.org/10.1094/PHYTO.1997.87.9.932

Zambrano, J. L., Francis, D. M., \& Redinbaugh, M. G. (2013). Identification of resistance to Maize rayado fino virus in maize inbred lines. Plant Dis., 97, 1418-1423. https://doi.org/10.1094/PDIS-01-13-0037-RE

Zambrano, J. L., Jones, M. W., Brenner, E., Francis, D. M., Tomas, A., \& Redinbaugh, M. G. (2014). Genetic analysis of resistance to six virus diseases in a multiple virus-resistant maize inbred line. Theor Appl Genet, 127, 867-880. https://doi.org/10.1007/s00122-014-2263-5

\section{Copyrights}

Copyright for this article is retained by the author(s), with first publication rights granted to the journal.

This is an open-access article distributed under the terms and conditions of the Creative Commons Attribution license (http://creativecommons.org/licenses/by/4.0/). 